Anne Besson

in Fantasmagories du Moyen Âge, sous la direction de Valérie Naudet et Elodie Burle, Publications de l’Université de Provence, Senefiance n`56, 2010, p. 27-35.

\title{
Usurper la médiévité : stratégies archaïsantes des réécritures arthuriennes contemporaines
}

Les réécritures arthuriennes contemporaines constituent un énorme corpus romanesque regroupant plusieurs dizaines de vastes cycles et de nombreux succès commerciaux, comme Les Dames du Lac de Marion Zimmer Bradley, les ensembles de Bernard Cornwell ou Stephen Lawhead - un corpus d'une grande homogénéité thématique (on y redit toujours la même histoire à peu près, avec les mêmes personnages à peu près), mais d'une identité générique en revanche hétérogène, fluctuant entre roman historique et fantasy.

Ce point ayant fait l'objet d'une précédente étude ${ }^{1}$, c'est un autre paradoxe qui nous retiendra ici, que signale le nom même sous lequel on regroupe ces textes et qui touche cette fois à leur rapport au temps. Réécritures affichant leur lien à une tradition intertextuelle médiévale, ces fictions n'en demeurent pas moins irréductiblement contemporaines. Leur ambition a beau consister à être reçues comme fidèles à une époque passée, à un Moyen Âge dont il s'agira même de rétablir une vérité déformée par la fiction, les obstacles se multiplient à l'évidence, génériques, thématiques et pragmatiques, qui rendent cet objectif a priori inaccessible : comment se faire passer pour plus vieux qu'on ne l'est?, et quelle légitimité pour un Moyen Âge ressuscité au travers d'un prisme si manifestement déformant?

Les réécritures arthuriennes devraient en effet se heurter au paradoxe : elles sont d'abord confrontées à leur propre statut, marginal, au sein du système littéraire contemporain (difficile semble-t-il de prétendre au sérieux historique ou même à l'inscription dans une intertextualité légitimante quand on est un sous-genre plutôt bâtard des littératures de grande diffusion quoique cela donne droit à une autre forme d'indulgence, on le verra). Mais nos réécritures ne peuvent pas non plus, de l'autre côté, se reposer sur ce que leurs sources, alléguées ou non, auraient pour leur part d'inattaquable, se rattacher à un statut solide qu'elles emprunteraient : elles savent au contraire se référer à un agrégat mobile de textes parfois contradictoires, et à des données historiques extrêmement lacunaires et imprégnées de légende; en outre, elles

\footnotetext{
${ }^{1}$ Anne Besson, «Le mythe culturel en fiction: deux relectures de la préhistoire arthurienne par les cycles de fantasy contemporains », dans le cadre du colloque «Lectures du Moyen-Age », 31 mars-2 avril 2005 , Université de Bretagne Sud (Lorient). In Images du Moyen Âge, sous la direction d'Isabelle Durand-Le Guern, PU de Rennes, «Interférences », 2006, p. 175-184. En ligne sur http://www.modernitesmedievales.org/articles/BessonFantasy.htm
} 
auront à intégrer, même dans les tentatives qui visent à reconstituer une chronologie médiévale «vraisemblable», un inexpugnable élément de merveilleux présent dans les sources.

Mais là encore, ces difficultés se renversent partiellement : les réécritures pourront alléguer justement la pluralité des sources pour justifier la version inédite supplémentaire de la même Matière arthurienne qu'elles proposent, tandis que l'un des attraits de cet ensemble de personnages et d'épisodes toujours repris tient précisément dans sa souplesse générique, entre histoire et légende, qui autorise un large éventail d'investissements possibles. Comment nos romans contemporains vont-ils donc exploiter les contraintes paradoxales qui pèsent sur eux ? Comment vont-ils s'y prendre pour convaincre leur lectorat, à rebours de toutes les évidences de leur contexte de production et de réception, de «suspendre son incrédulité » historique pour accepter de se croire face à un texte issu des temps médiévaux ? Ils prétendent d'abord dire le Moyen Âge dès lors qu'ils répètent l'histoire d'Arthur : que ce soit dans les genres du roman historique ou de la fantasy, les réécritures revendiquent l'existence de sources auxquelles elles se confrontent comme opérant autant de « retours aux sources ». Mais elles usurpent aussi, au service d'un même objectif, le temps qui nous sépare du Moyen Âge, son ancienneté - comme si les volumes provenaient eux-mêmes du passé, y ayant été écrits : c'est l'épaisseur même de la durée qui se trouve ultimement visée.

La nature et la visée de nos textes consistent à présenter une version nouvelle des éléments disponibles quant à la légendaire histoire d'Arthur. Ils s'en autorisent d'un argument selon lequel ils ne feraient que prolonger une tendance médiévale bien attestée, puisque la matière romanesque arthurienne a fait l'objet d'une intense circulation entre les manuscrits dès les origines, et au fil du Moyen Âge d'une impressionnante pulsion d'expansion, une «tendance à la cyclisation » selon Zumthor ${ }^{2}$. Cependant, par ce renvoi quasi-systématique à la prolifération des sources, les réécritures contemporaines esquivent aussi, et en même temps, la question de savoir à quelle tradition précisément elles se rattachent, et même en fait à quel Moyen Âge elles choisissent de renvoyer. La légende arthurienne telle qu'elle nous ait parvenus en convoque et en synthétise en effet plusieurs : le contexte historique est celui des fin $\mathrm{V}^{\mathrm{e}}$-début $\mathrm{VI}^{\mathrm{e}}$ siècle anglais, où l'influence romaine décline au profit de chefs de clans, de chefs de guerre comme peut-être cet Arthur qui combattit à Badon et à Camlann. Mais le personnage n'accède à l'existence romanesque que bien plus tard, dans un contexte qui se

\footnotetext{
${ }^{2}$ Paul Zumthor, Essai de poétique médiévale, Paris, Seuil «Poétique », 1972, p. 367.
} 
l'approprie : les ouvrages de Chrétien de Troyes évoquent, autour de la cour arthurienne, le conflit ou la coexistence de valeurs qui sont celles de leur période d'écriture, la fin du XII siècle, qui se trouve ainsi fournir un autre fond chronologique possible pour les réécritures ; on a pu faire les mêmes analyses au sujet de l'œuvre en prose de Thomas Malory, qui insuffle par exemple dans sa reprise du $\mathrm{XV}^{\mathrm{e}}$ la mode de la chevalerie que connaît son époque ${ }^{3}$.

Diverses stratégies de positionnement chronologique sont donc possibles: les âges sombres des $\mathrm{V}^{\mathrm{e}}-\mathrm{VI}^{\mathrm{e}}$ siècles inspireront davantage les réécritures visant l'historicité et se rattachant donc au roman historique (Rosemary Sutcliff, Michel Rio, Bernard Cornwell et bien d'autres ${ }^{4}$ ), tandis que l'image plus conforme aux souvenirs scolaires et aux canons «moyenâgeux » des cours des $\mathrm{XII}^{\mathrm{e}}-\mathrm{XIII}{ }^{\mathrm{e}}$ s., avec leurs chevaliers et leurs gentes dames, correspondra davantage aux reprises empruntant la voie d'un merveilleux qui s'y trouve spontanément associé. La première de ces options est en général affichée, comme chez Michel Rio, qui date les chapitres du volume central de sa trilogie, Morgane, et propose en outre en annexe un tableau récapitulatif d'une chronologie reconstituée avec un soin d'historien. Dans la veine merveilleuse en revanche, les choix de datation demeurent le plus souvent implicites, voire volontairement brouillés : même en mettant à part une entreprise comme celle de JeanLouis Fetjaine, qui imagine une pré-histoire de la geste arthurienne dans un passé reculé où cohabitaient sur Terre différentes races (les quatre Tribus de Dana: hommes, elfes, nains et monstres), une œuvre plus « canonique », comme celle de Marion Zimmer Bradley, n'est pas sans évoquer sur ce point les «brumes » de son titre.

Dans ses «Remerciements $»^{5}$, la romancière commence, bien entendu, par relever la pluralité des sources («difficile, tant elles sont nombreuses, de dresser la liste exhaustive des sources ayant servi à l'élaboration de cet ouvrage »). Elle va insister sur des références historiques ou pseudo-historiques, comme les travaux d'archéologie sur les sites « arthuriens » menés par Geoffrey Ashe (une référence d'ailleurs également alléguée par Rio), et sur un type d'érudition plus ésotérique concernant l'histoire des religions et les résurgences « païennes » contemporaines. Autant d'éléments, sites de fouilles et origines du christianisme, qui pointent vers l'option chronologique la plus reculée, mais que vient contrebalancer l'évidente source littéraire de Bradley, qu'elle ne cite pas dans les « Remerciements » pour y renvoyer autrement, par le biais de l'épigraphe qui lui est empruntée : Le Morte Darthur de

\footnotetext{
${ }^{3}$ Helen Cooper évoque ce «cult of chivalry » dans son introduction de Le Morte Darthur de Thomas Malory (The Winchester Manuscript, Oxford U.Press, « Oxford World's Classics », 1998).

${ }^{4}$ Voir sur ce corpus l'ouvrage de Marc Rolland, Arthur, un mythe hérö̈que au XXe siècle, Presses Universitaires de Rennes, «Interférences », 2004.

${ }^{5}$ Marion Zimmer Bradley, Les Dames du Lac, Livre de Poche, 2002, p. 7-9.
} 
Thomas Malory, dont la romancière va suivre souvent scrupuleusement la version, et à laquelle elle se réfère encore quand il s'agit d'imposer la sienne propre. Mais avec Malory, c'est la cour chrétienne, ses chevaliers et ses merveilles, qui font retour, dans une version tardive qui plus est... Egalement évasive sur la vaste tradition convoquée pour sa «trilogie celtique » consacrée à Gwalchmai-Gauvain, «inspirée tout d'abord de plusieurs sources celtiques, puis, dans un second temps, de tous les récits écrits à ce jour à propos d'Arthur ${ }^{6}$ (pas moins !), Gillian Bradshaw prend quant à elle, celtisme oblige, plus résolument position aux $\mathrm{V}^{\mathrm{e}}-\mathrm{VI}^{\mathrm{e}}$ siècles, mais cet ancrage demeure menacé de brouillages, par la franche inscription du texte dans le genre de la fantasy d'abord, par la nécessaire prudence que doivent inspirer les appels aux « sources celtes » ensuite ${ }^{7}$.

Il est ainsi, bien souvent, délicat de tirer des éléments de contextualisation historique et intertextuelle fiables du péritexte pseudo-érudit qui entoure les réécritures arthuriennes, si bien qu'on est amené à y voir avant tout sa propre justification. Ces textes d'accompagnement, en introduction ou en clôture des ouvrages, ne sont vraiment là que pour rappeler au lecteur qu'une ou, mieux, des traditions existent, et que les auteurs en ont connaissance. Il s'agit de citer des sources, de dire à la fois la consultation d'innombrables garants et la liberté prise à leur égard, en une stratégie d'affichage de l'érudition qui trouve sa forme achevée dans la bibliographie. Une telle liste d'ouvrages, qu'on nous présente comme consultés par l'auteur et proposés au lecteur pour son édification personnelle, figure par exemple à la fin des volumes de la «Trilogie des Elfes» de Jean-Louis Fetjaine et dans sa suite, Le Pas de Merlin. En même temps que ce cycle, nettement «médiéval-fantastique », n'hésite pas à s'infléchir vers le roman historique, il nous renvoie à un mélange, représentatif des sources alléguées dans ce type de contexte, d'ouvrages de synthèse rigoureux, mais aussi de moins sérieux représentants du celtisme français, comme l'inévitable Jean Markale ${ }^{8}$.

Les références intertextuelles peuvent enfin se faire depuis l'intérieur des textes, qui intègrent des citations de leurs sources littéraires: c'est encore le cas chez Jean-Louis Fetjaine, qui fait figurer, en italiques, de longs passages du Lancelot $d u$ Lac (13 ${ }^{\text {ème }}$, Fracs Mosès) qu'il intègre à son développement, quitte à brouiller un peu plus son ancrage atemporel $^{9}$. Mais ce sont plus souvent les fameuses «sources celtiques », récits

\footnotetext{
${ }^{6}$ Gillian Bradshaw, Hawk of May (1980), Faucon de Mai, Aix-en-Provence, Nestiveqnen, 2004, p. 5.

${ }^{7}$ Voir l'article d'Alexis Léonard, «Le Moyen Âge sacrifié : le retour des celtes », in Images du Moyen Âge, op. cit., p. 57-67.

${ }^{8}$ Je renvoie encore aux travaux d'Alexis Léonard, ainsi qu'à la synthèse de Florence Plet sur la BD arthurienne, où elle met en lumière chez les scénaristes le recours à ces mêmes références (in Arthur au miroir du temps, ouvrage collectif sous la direction d'A. Besson, à paraître chez Terre de Brume).

${ }^{9}$ L'Heure des Elfes (2000), Paris, Pocket SF, 2002, p. 50 et 77-79.
} 
mythologiques et poèmes à la datation incertaine, qui se trouvent ainsi exploitées sous forme de reprises dans le corps des textes. Stephen Lawhead fait notamment un usage systématique des Mabinogions ${ }^{10}$, dont les différents récits participent à la trame romanesque comme « récits dans le récit », racontés lors de veillées ou de banquets ${ }^{11}$. Gillian Bradshaw «adapte » quant à elle des poèmes irlandais dont elle précise allusivement les références dans son «Avant-propos » ${ }^{12}$, soulignant du moins leur véritable datation et leur caractère anachronique, tandis que Lawhead cite encore, sans autre précaution, les poèmes attribués au barde du VI ${ }^{\mathrm{e}}$ siècle Taliesin. Celui-ci devient en effet un des personnages principaux de son cycle, le héros éponyme du premier volume, avant Merlin, donné ici comme son fils ; Taliesin figure également comme personnage dans le cycle de Bradley, ou se voit encore cité dans la trilogie de G. G. Kay, «La Tapisserie de Fionavar », par un immortel dont le poète fut l'une des incarnations ${ }^{13}$. Le choix de nos romans contemporains se porte donc significativement moins sur Merlin, pourtant plus fameux, que sur Taliesin, à la fois source intertextuelle suspecte, dont les poèmes allusifs (cette allure cryptique se faisant gage d'archaïsme) nous deviennent pourtant familiers tant se multiplient les réécritures qui s'en emparent, et personnage fondateur, qu'il soit le père de Merlin, son prédécesseur, son ancienne incarnation - son annonciateur toujours, comme Merlin le sera à son tour d'Arthur, et sa « version celtique » pour tout dire.

On peut voir, dans cette substitution de personnages, une manifestation de la stratégie générale des réécritures arthuriennes vis-à-vis de leurs sources mâtinées d'histoire et de fiction. Elle consiste en effet à opérer sur elles et par rapport à elles un retour, conforme au grand mouvement de remontée aux origines, d'expansion vers l'arrière, qui caractérise les néo-médiévalismes : retour sur les sources, dans le but de les corriger pour rétablir une vérité déformée, retour aux sources, qui remonte en deçà des savoirs constitués pour viser comme un substrat culturel, qui prend souvent la forme du paganisme celtique ou d'une magie immémoriale. Marion Zimmer Bradley, grâce à son double système de références, peut

\footnotetext{
${ }^{10}$ Les Quatre Branches du Mabinogi et autres contes gallois du Moyen Âge, éd. et trad. Pierre-Yves Lambert, Paris, Gallimard, « L'aube des peuples », 1993.

${ }^{11}$ Dans le premier volume, Taliesin (Paris, Buchet-Castel, 1997), «l'histoire de Pwyll, prince d'Annwfn », p. 94-104 («et ainsi se termine cette branche du Mabinogi ») et «l'histoire de Math ap Mathonwy », évoquée p. 240 ; dans Merlin (Paris, Buchet-Castel, 1997), «le Mabinogi de Manawyddan », p. 173-183; dans Arthur (Paris, Buchet-Castel, 1997), une «branche du Mabinogi », «Bronwen, fille de Llyr », p. 275-288, « le songe de Rhonabwy », «conte celte », p. 379-388 et p. 402, un récit juste évoqué par son titre, «La vision de Taliesin, également nommé La chanson du Royaume de l'Eté ».

${ }^{12}$ Faucon de Mai, op. cit., p. 5-6.

${ }^{13}$ L'Arbre de l'été (Paris, Pygmalion, 1996), p. 346, ou La Voie obscure (Paris, Pygmalion, 1997), p. 24-25 pour une citation du Preideu Annwvn, le poème sans doute le plus fameux de Taliesin, qui conte les voyages du navire Prydwen.
} 
astucieusement combiner les deux faces de cette stratégie, retour sur les sources et donc retour aux sources : d'une part, elle emprunte des épisodes entiers à Malory, en les présentant toutefois comme le compte-rendu vécu de Morgane, ce qui permet de corriger des détails présentés comme invraisemblables et mis sur le compte de la déformation légendaire ${ }^{14}$. Se donnant ainsi comme plus véritable que le texte sur lequel elle s'appuie (et dont elle a déjà omis de dire qu'il était tardif), s'appuyant par ailleurs sur les sources plus « authentiques » et «archaïques » que nous avons mentionnées, elle peut réinventer cette Morgane, personnage tout à fait secondaire, et parfaitement maléfique, chez Malory, en avatar de la Déesse-mère s'unissant cycliquement à une incarnation du Dieu Cornu, dans le cadre, promis au plus grand succès, d'un imaginaire du pré-christianisme comme refuge d'une puissance féminine et naturelle.

Pour donner l'impression de remonter ainsi dans le temps, le retour aux sources onomastiques constitue un des procédés les plus courants des réécritures celtisantes : que leur préférence aille plutôt vers un contexte pseudo-historique ou mythologique, dans tous les cas elles vont «revenir» aux versions galloises des noms de lieux et de personnages, pour un effet archaïsant garanti vu leur exotisme orthographique. Ainsi, Gwalchmaï remplace Gauvain, dans le cycle historique de Rosemary Sutcliff comme dans celui, fantastique, de Gillian Bradshaw, et se voit préférer la graphie Gwalcmai chez Lawhead. Merlin devient Myrddin aussi bien dans le roman historique Porius de John Cowper Powys que dans l'elfique trilogie de Fetjaine. Guenièvre/Guinevere suscite davantage de créativité dans les variantes: Gwinfreda chez Gloag, Guenhumara chez Sutcliff, Gwynhwaevar chez Mary Stewart, Gwenhwyvar chez Lawhead. On pourrait multiplier les exemples, où s'étalent à plaisir successions de consonnes, « $\mathrm{W} », \ll \mathrm{h} »$, double « $\mathrm{ll} »$ et autres chuintantes, si bien que Gillian Bradshaw, qui propose aussi une carte de la Grande-Bretagne aux toponymes celtiques, peut compléter son exposé des sources par un petit précis de prononciation du gallois.

Ces graphies (presque) imprononçables se donnent comme des gages d'authenticité archaïque, mais l'héritage du passé subit au passage, il faut le noter, un impressionnant écrasement chronologique : pour être superposable au présent, à la façon de deux feuilles de papier, l'épaisseur de ses strates doit au préalable se trouver aplatie. Les variantes orthographiques qui, au Moyen Âge, résultaient de divergences dialectales ou d'évolutions

\footnotetext{
${ }^{14}$ Voir ma rapide analyse d'un tel procédé dans Les Brumes d'Avalon (op.cit., p. 254-255), dans « Le mythe culturel en fiction : deux relectures de la préhistoire arthurienne par la fantasy contemporaine », article cité, p. 179.
} 
linguistiques séculaires, se muent ainsi en choix d'options disponibles, auxquelles les réécritures arthuriennes ont accès simultanément, dans une synchronie instantanée.

Quand un cycle ne se contente plus d'adopter une fois pour toutes une orthographe archaïsante, mais intègre la déformation onomastique à son propre développement volumique, on passe à un type de reprise structurelle où la forme même du texte mime l'éloignement temporel et contribue à l'écrasement chronologique qui résulte de la capacité ainsi postulée à être d'un coup, très vieux. Il faut d'abord reconnaître que beaucoup de nos textes font preuve d'une inventivité formelle assez limitée dans leurs procédés de narration ou techniques d'écriture : c'est ainsi que la plupart des réécritures affichent un peu maladroitement leur caractère contemporain au travers d'une narration à la première personne très majoritaire. Il s'agit d'élire un témoin privilégié pour varier l'angle de vue sur la même éternelle histoire, tout en prétendant à l'authenticité du compte-rendu vécu et à sa supériorité sur les légendes colportées (les sources); quasiment tous les personnages arthuriens ont eu ce douteux honneur, Morgane bien sûr chez Bradley mais également dans les cycles de Fay Sampson ou Nancy Springer, qui donne également la parole à Mordred, Guenièvre par exemple dans les deux romans de Nancy McKenzie, Guenièvre, l'enfant-reine et Guenièvre, la reine de Bretagne, Gauvain/Gwalchmai pour Bradshaw, ou encore Merlin dans le volume éponyme de Lawhead et dans la trilogie de Mary Stewart. Dans ce dernier cas, et plus encore dans celui d'Arthur, qui raconte sa propre histoire dans Sword at Sunset de Rosemary Sutcliff, le gain d'un point de vue privilégié se paie en revanche, comme le relève Marc Rolland, d'une franche invraisemblance des modalités de rédaction (entre deux batailles ? après la mort ?). C'est pourquoi des personnages secondaires ou inédits sont souvent choisis pour témoins, narrateurs anonymes d'une histoire objective, à l'image de Derfel, guerrier devenu saint, dans Le Roi de l'hiver, premier volume du cycle de Bernard Cornwell.

La volonté de conférer une historicité plausible (ou une authenticité usurpée...) au témoignage fictionnel refait explicitement surface dans un roman historique de 1996, l'intéressante Arthuriad de Frederick Lees ${ }^{15}$. Le texte présente un autre type de préface pseudo-érudite que celles que nous avons rencontrées jusqu'ici, qui n'abdiquaient jamais un sérieux un peu naïf et surtout n'appartenaient pas à la fiction mais lui conféraient une légimité «extérieure » en renseignant sur ses origines. Lees au contraire intègre sa préface à la construction d'une vraisemblance fictionnelle pour son roman, en y présentant l'enquête

\footnotetext{
${ }^{15}$ Merci à Marc Rolland qui m'a permis de découvrir cette œuvre mal connue en France.
} 
historiographique du «traducteur » qui édite le texte, et reconstitue comment ce manuscrit trouvé de nos jours en Egypte est parvenu jusqu'à nous... Le procédé, très classique, relève cependant moins ici de l'habituel artifice de voilement/dévoilement de la fiction que d'une stratégie archaïsante encore une fois : il justifie le déplacement instantané du texte médiéval dans notre présent, et matérialise ce caractère de « mise à jour » du passé que veut, dans tous les sens de l'expression, présenter la réécriture, à travers l'image même de l'exhumation du manuscrit.

Bradley esquisse un autre procédé au riche potentiel, qui exploite cette fois la structure du cycle au service du même objectif, l'accès immédiat à l'histoire reculée, nimbée de légende sacrée. On sait que le recours au cycle est généralisé, dans les littératures de genre et de grande diffusion ${ }^{16}$, et les réécritures arthuriennes ont d'autant moins de raison de s'en priver que le modèle des cycles médiévaux, qui en annoncent souvent déjà des tendances toujours à l'œuvre aujourd'hui, les y autorisent et même les y invitent dans leur quête imitative. Le cycle contemporain a beau reposer pour sa définition sur une pluralité de volumes chronologiquement reliés, critère éditorial qui n'a pas d'équivalent dans les manuscrits médiévaux, le choix récurrent du terme de « cycle », par les auteurs et éditeurs, pour désigner les ensembles néo-arthuriens, fait clairement signe vers ce modèle, même s'il demeure presque toujours implicite.

Bradley tire quant à elle un profit original de cette succession de volumes discontinus qui définit le cycle aujourd'hui, en l'égalant frauduleusement à la succession longue des cycles arthuriens médiévaux. Après les deux volumes du roman Les Dames du Lac, le troisième, Le Secret d'Avalon, présente cette nouvelle tentative d'usurpation d'une marque de médiévité qui n'est autre que le fruit même de ce temps long, dont la contemporanéité tente si désespérément de forcer l'accès. Le Secret d'Avalon, prequel ou «antépisode » des Dames du $L a c$, fait le lien entre celles-ci et un roman «romain» de Bradley, La Colline du dernier adieu; en trois parties et trois personnages féminins, le volume balaie plus de trois siècles d'histoire. Pièce maîtresse de l'édifice chronologique du cycle, il consolide d'emblée sa volonté de se doter d'un caractère fondateur, avec, en ouverture, plusieurs indexs, dessins et cartes - ancrage pseudo-documentaire, puis un prologue cosmogonique sur la création d'Avalon - ancrage mythique bien sûr. A tout cela s'ajoute une « note liminaire », semble-t-il éditoriale, qui souligne d'abondance les rapprochements structuraux à la tradition médiévale : cet épisode de genèse «donne son sens et son unité à la fiction épique de Marion Zimmer

\footnotetext{
${ }^{16}$ Voir Anne Besson, D’Asimov à Tolkien, cycles et séries en littérature de genre, CNRS Editions, « Littérature », 2004.
} 
Bradley, dans le droit fil de nos textes médiévaux », cet «univers chevaleresque dont notre littérature courtoise a su éterniser les traits [...] dans le temps immobile ou cyclique des grands mythes ». Sur le modèle de la translation du Graal en Angleterre par Joseph d'Arimathie, « [i]l fallait donc rattacher le Temps des Aventures et de la Chevalerie au Temps de l'évangélisation et de la conquête romaine, sous peine de mutiler la tradition ${ }^{17}$.

Enfin, belle astuce, la note prévient de divergences onomastiques entre ce roman et les précédents : les personnages récurrents ne portent plus tout à fait le même nom, Gavain et Kellen, dans ce nouveau roman donné comme plus ancien, s'appellent Gawen et Caillean, Igraine Ygerne, «conformément aux usages du temps », conclue la note ${ }^{18}$. Les déformations orthographiques sont ainsi alléguées en exemples alors qu'elles constituent bien plus le seul fondement à la prétention de ce texte, parce qu'il remonte dans le temps de l'intrigue, à être plus vieux encore que les autres. Et comment mieux faire semblant qu'en reproduisant ce recul déformant que seul permet normalement le passage des siècles ?

Nous avons vu se déployer un éventail de tactiques, au service d'un objectif paradoxal, qui peut sembler presque absurde : faire semblant d'être «du» Moyen Âge, dans une double acception du morphème « de », partitif et indiquant l'origine spatio-temporelle - un morceau de passé, magiquement déplacé de ce contexte. Il faut alors se demander pourquoi ?, ou à quoi bon ?, et c'est donc du côté de l'appréhension du genre par son lectorat que nous aboutirons : il appartient au contrat ultra-codifié du genre que les auteurs fournissent des indices d'archaïsme, de médiévité ; au public, ne reste qu'à se laisser convaincre. Le lectorat de ces corpus, entre fantasy et roman historique, ne manque à l'évidence pas de ce qu'on peut considérer comme une indulgence paresseuse ou comme une capacité d'immersion fictionnelle sur-développée - un fort désir de croire, qui constitue bien entendu, et mieux que toutes les stratégies, le plus sûr moyen de transport pour ce voyage dans le temps qui ramène le Moyen Âge aujourd'hui. Même si le vraisemblable obtenu peut sembler plus ou moins consistant ou convaincant, la seule mission du texte n'est, jamais, que de favoriser ce transport volontaire, ce désir de Moyen Âge, qui suffit à le susciter et à le ressusciter.

\section{Bibliographie du corpus romanesque :}

\footnotetext{
${ }^{17}$ Le Secret d'Avalon, Paris, Editions de Fallois, 1997, p. 7. Je souligne, parmi les nombreuses expressions qui le mériteraient, la formule où le phénomène d'usurpation du temps long se fait le plus frappant : la «tradition »en question n'est autre ici en effet que celle qui se constitue sous nos yeux.

${ }^{18}$ Ibid., p. 8.
} 
Bradley, Marion Zimmer : The Mists of Avalon (1982) ; trad. Brigitte Chabrol en deux tomes, Paris, Pygmalion-Gérard Watelet : Les Dames du Lac, 1986, Livre de Poche, 2002, Les Brumes d'Avalon, 1987, Livre de Poche, 2003.

The Lady of Avalon (1997), Le Secret d'Avalon, trad. Jean Esch, Paris, Editions de Fallois, 1997, Livre de Poche, 2003.

En collaboration avec Diana L. Paxson, The Priestess of Avalon (2000), La Prêtresse d'Avalon, trad. Monique Lebailly et Edith Ochs, Paris, Editions du Rocher, 2001, J'ai Lu SF, 2003.

The Ancestors of Avalon (2004), Les Ancêtres d'Avalon, trad. Pascale Renaud-Grobras, Paris, Editions du Rocher, 2005.

Bradshaw, Gillian : Hawk of May (1980), Faucon de Mai, trad. Arnaud Boulin, Aix-en-Provence, Nestiveqnen, 2004.

Kingdom of Summer, New York, Simon and Schuster, 1981.

Cornwell, Bernard : «The Warlord Chronicles », Paris, Editions de Fallois :

The Winter King (1996), Le Roi de l'hiver, trad. Pierre-Emmanuel Dauzat, 1998.

Enemy of God (1997), L'Ennemi de Dieu, trad. Pierre-Emmanuel Dauzat, 2000.

Excalibur (1998), trad. Monique et Hugues Lebailly, 2001.

Fetjaine, Jean-Louis : Le Crépuscule des Elfes, Paris, Belfond, 1998, Pocket SF, 2002

La Nuit des Elfes, Paris, Belfond, 1999, Pocket SF, 2002.

L'Heure des elfes, Paris, Belfond, 2000, Pocket SF, 2002.

Le Pas de Merlin, Paris, Belfond, 2002.

Brocéliande, Paris, Belfond, 2004.

Gloag, John : Artorius Rex, New York, Saint Martin’s Press, 1977.

Kay, Guy Gavriel : «La Tapisserie de Fionavar », trad. Elizabeth Vonaburg :

The Summer Tree (1984), L'Arbre de l'été, Québec, Ed. Québec Amérique, 1994, Paris, Pygmalion, 1996, J'ai Lu, 1998.

The wandering Fire (1986), Le Feu vagabond, Québec, Ed. Québec Amérique, 1994, Paris, Pygmalion, 1996, J'ai Lu, 1998.

The darkest Road (1986), La Voie obscure, Québec, Ed. Québec Amérique, 1995, Paris, Pygmalion, 1997, J'ai Lu, 1999.

Lawhead, Stephen : «Pendragon », trad. Luc Carissimo, Paris, Buchet-Castel :

Taliesin (1987), 1997.

Merlin (1987), 1997.

Arthur (1987), 1997.

Pendragon (1987), 1998.

Grail (1997), Le Graal, 1999.

et, hors cycle : Avalon, the Return of King Arthur (1999), Avalon, le retour du roi Arthur, trad. Emmanuèle de Lesseps, Paris, Buchet-Castel, 2001.

Lees, Frederick : The Arthuriad of Catumandus, Hong Kong et Londres, Crane Books, 1996.

MacKenzie, Nancy : The Child Queen (1994), Guenièvre, l'enfant-reine, trad. B. J. Willis, Paris, Le Pré-auxClercs, 2002.

The high Queen (1995), Guenièvre, la reine de Bretagne, trad. François Truchaud, Paris, Le Pré-aux-Clercs, 2003.

Powys, John Cowper : Porius, Londres, Village Press, 1974.

Rio, Michel : Merlin, Morgane, Arthur, Paris, Seuil, 1989, 1999 et 2001.

Sampson, Fay : «Daughter of Tintagel », Londres, Headline, rééd. Cosmos, 2005.

Wise Woman's Telling, 1989,

White Nun's Telling, 1989,

Blacksmith's Telling, 1990, 
Taliesin's Telling, 1991,

Herself, 1992.

Springer, Nancy : «Tales from Camelot »

I am Mordred, New York, Philomel, 1998.

I am Morgan le Fay, New York, Philomel, 2001.

\section{Stewart, Mary :}

The Crystal Cave (1970), La Grotte de cristal, trad. Brigitte Amiot, Paris, Calmann-Lévy, 2006.

The hollow hills, Londres, Hodder and Stoughton, 1973.

The last enchantment, Londres, Hodder and Stoughton, 1977.

The wicked day, Londres, Hodder and Stoughton, 1983.

Sutcliff, Rosemary : Sword at Sunset, New York, Coward-McCann, Londres, Hodder and Stoughton, 1963. 